\title{
CHRONIQUE
}

\section{Livre sterling pour livre sterling.}

Au mois de juin I9I9, le Gouvernement britannique a pris une décision qui n'a peut-être pas assez été remarquée dans les autres pays, c'est celle de doubler les sommes recueillies par la charité privée en faveur des œuvres de secours.

Le principe "Livre sterling pour Livre sterling 》, auquel la Revue $^{1}$ a précédemment fait allusion, a reçu son application dans des proportions tout à fait remarquables.

En réponse à une question posée à la Chambre des Communes, le 17 mai I920, Mr. Baldwin a fait connaître le chiffre des allocations faites par le Trésor suivant les règles qui avaient été fixées l'année précédente.

I6 sociétés $^{2}$ ont reçu des allocations variant de I,000 à I 20,000 lst. pour un total de 452,329 lst.

Parmi les sociétés bénéficiaires de ces allocations, on relève la Croix-Rouge britannique 30,000 lst., la branche anglaise de la Croix-Rouge I8,00o lst., le fonds de la Croix-Rouge arménienne 5,I94 lst. L'allocation la plus forte a été celle du fonds de secours d'urgence pour Vienne $\mathrm{I}_{20}, \mathrm{I}_{4} \mathrm{I}$ lst.

Ces chiffres sont édifiants. Ils témoignent de l'important effort du Gouvernement britannique pour soutenir les initiatives charitables du Royaume-Uni, et pour encourager par là même les donateurs à se montrer généreux.

1 Voy. Revue, I5 avril I920, p. 393.

2 British Red Cross Society............................... 30,00

Anglo-Czech Relief Fund............ » $\mathrm{r}_{7,565}$

Friends' War Victims' Relief

Committee (Poland).............. " I6,66I

Friends' Emergency Committee (Vienna).... " 64,567

Vienna Emergency Relief Fund.......... ) I20,I4I

Serbian Red Cross Society............. " I 8,000

Serbian Relief Fund.............. " 13,336

Scottish Women's Hospitals (Serbia)....... "

Action Lodge Famine Relief Fund (Budapest).. " " $\quad I_{4,904}$ 


\section{CHRONIQUE Livre pour livre.}

La communication de Mr. Baldwin fait connaître la procédure suivie pour ces allocations.

Un comité officiel pour le secours en Europe a été constitué. Ses membres étaient à la date du r5 mai I920:

Sir Melvill Beachcroft, président; MM. Arthur Franklin, Percy Strauss, Arthur Prideaux, "Central Trustees of Controlled War Charities"; Sir Cyril K. Butler, K. B. E. ; Sir William Goode "British Director of Relief》, ex-officio; Mrs J. R. Brooke (Miss D. Lamb), secrétaire; Miss I. Newson, secrétaireadjointe.

Ce comité ${ }^{1}$ examine toutes les demandes qui lui sont adressées informe le Trésor du montant des allocations demandées et du nom des sociétés appelées à en bénéficier. En faisant leur demande, les sociétés sont tenues de justifier de leur enregistrement régulier au "War Charities" Act ", de fournir des renseignements concernant leur comité, leurs membres et leur activité en général, ainsi qu'un budget et un compte rendu de l'emploi des allocations antérieures qui auraient pu leur être faites.

Des allocations ont été refusées pour les causes suivantes :

$I^{\circ}$ Les dépenses effectuées ne correspondaient pas aux principes de secours adoptés par le Comité officiel ${ }^{2} .2^{0}$. Les sociétés demandant des allocations ne remplissaient pas les conditions exigées par le Comité. $3^{\circ}$ Le budget soumis par une société accordait une part trop élevée aux frais d'administration au détriment des secours. $4^{\circ}$ Les comptes présentés étaient insuffisants.

Westminster Catholic Federation.......... » $\quad 2,588$

Montenegrin Relief Fund.............. » I,486

Fund in Aid for Jewish Victims of the War

(Poland).............. "74,825

Armenian Refugees' (Lord Mayor's) Fund..... » 27,8 3

Armenian Refugees' Clothing Fund......... " 34,933

Armenian Red Cross Fund.............. " 5, r94

Syria and Palestine Relief Fund........., , 2,000

1 "Official Committee for Relief in Europe, room 530, Palace Chambers, S. W. I."

${ }^{2}$ Le Gouvernement britannique ne double pas les sommes dépensées en faveur de l'Allemagne. 


\section{CHRONIQUE}

\section{Livre pour livre.}

On ne saurait trop insister sur le haut intérêt de cette allocation dite livre sterling pour livre sterling. Il serait à souhaiter que d'autres gouvernements suivent le Gouvernement britannique dans cette voie.

Si dans tous les pays donateurs, les ouvres de secours s'exerçant en faveur de l'étranger voyaient leurs ressources doublées ipso facto par leur gouvernement, franc pour franc, dollar pour dollar, etc. il n'est pas douteux que l'entr'aide internationale recevrait une impulsion nouvelle pour le plus grand bien des populations éprouvées au lendemain du conflit mondial.

E. C.

\section{L'Etat et les victimes de la guerre en Bulgarie.}

Les souffrances accumulées par les trois guerres successives entreprises par le peuple bulgare pour parfaire son unité nationale sont si étendues, si multiples, que la charité publique et privée, débordée, se trouve absolument impuissante à en réduire les effets douloureux sans le concours de l'Etat. La Bulgarie compte à l'heure actuelle 270,000 orphelins et 70,000 mutilés et invalides. La guerre finie, il s'agit de commencer "une autre guerre", organisée et dirigée contre les innombrables fléaux engendrés par la lutte meurtrière qui vient de prendre fin. En Bulgarie la conduite de cette nouvelle guerre est assumée par l'Etat qui est en train d'élaborer une série de dispositions tendant à grouper et à intensifier les effets épars de la charité privée et publique dans le pays, et à en coordonner l'action bienfaisante.

L'organe central de cette action concentrée sera la Direction pour l'assistance aux victimes de la guerre, une institution ayant pour mission de réunir et de diriger rationnellement tous les efforts en vue de la protection morale et matérielle des victimes de la guerre. La création de cet organe est prévue dans le projet de loi spécial à ce sujet, œuvre du président du Conseil, M. Stamboliisky. Voici les grandes lignes de ce projet : 